\title{
Preparation and characterization of Co/PAN-based carbon fibrous composites
}

\author{
Z.X. Jiang ${ }^{1}$, Y.Y. Yao ${ }^{2, a}$, and G. Jiang ${ }^{1}$ \\ ${ }^{1}$ Institute of Atomic and Molecular Physics, Sichuan University, Chengdu 610065, P.R. China \\ 2 Textile College, Sichuan University, Chengdu 610065, P.R. China
}

Accepted: 5 September 2011

Published online: 28 September 2011 - (C) EDP Sciences 2011

Eur. Phys. J. Appl. Phys. 55, 10404 (2011), DOI: 10.1051/epjap/2011100503

An error occurred during the publication process in the Aims and Scopes sections of the contents: This article should be under section Nanomaterials and Nanotechnologies.

The author names have been misspelt and should read as above.

We apologize for this inconvenience.

\footnotetext{
${ }^{a}$ e-mail: yongyiyao@scu.edu.cn
} 